\title{
Hospitais gerais: inserção nas redes de atenção à saúde e fatores condicionantes de sua atuação
}

\author{
General hospitals: inclusion in the healthcare networks, and factors \\ that determine their performance
}

Fabiane Gorni Borsato (https://orcid.org/0000-0002-2977-1963) ${ }^{1}$

Brígida Gimenez Carvalho (https://orcid.org/0000-0003-3850-870X) ${ }^{1}$

${ }^{1}$ Programa de PósGraduação em Saúde Coletiva, Universidade Estadual de Londrina. Rodovia Celso Garcia Cid PR-445/Km 380, Campus Universitário. 86057-970 Londrina PR Brasil. fgborsato@gmail.com

\begin{abstract}
The scope of this study was to analyze the inclusion of general hospitals in Healthcare Networks and the factors that determine their participation in these networks. A \& quot;scoping reviewerquot; was conducted with the selection of 14 articles published between January 2011 and June 2018. From the mapping of the results the categories of analysis were constituted: Hospital, regionalization and economy of scale; Fragility of network organization and hospital as first choice; and Hospital and evidence of (dis) integration of the Healthcare Networks. The data revealed factors that determine the performance of the hospitals in the networks, highlighting the inadequate distribution of these services and their levels of scale in the territories; a considerable number of small-scale hospitals; the weaknesses of the organization of networked services, with little integration between the healthcare outlets; and the hegemony of the biomedical model. The conclusion drawn is that the optimal inclusion of general hospitals in Healthcare Networks will depend on the resolution of challenges related to the planning and implementation of actions in the various components of Healthcare Networks, regional availability of health services, improvement of regulatory mechanisms and of communication between the services.
\end{abstract}

Key words Hospitals, Regionalization, Comprehensive healthcare, Public health.
Resumo Este estudo objetivou analisar a inserção dos hospitais gerais nas Redes de Atenção à Saúde e os fatores condicionantes de seu modo de participação nas mesmas. Realizou-se um "scoping review", com a seleção de 14 artigos publicados de janeiro de 2011 a junho de 2018. Com o mapeamento dos resultados constituiu-se as categorias de análise: Hospital, regionalização e economia de escala, Fragilidade de organização da rede e hospitais como primeira escolha, e Hospital e evidências de (des)integração da Redes de Atenção à Saúde. Os dados mostraram fatores que condicionam a maneira como os Hospitais atuam nas RAS, tendo destaque a inadequada distribuição destes serviços e de seus níveis de escala nos territórios; um número considerável de Hospitais de Pequeno Porte; as fragilidades da organização dos serviços em rede, com pouca articulação entre os pontos de atenção; e a hegemonia do modelo biomédico. Concluiu-se que a melhor inserção dos hospitais gerais nas redes dependerá do enfrentamento de desafios relacionados ao planejamento $e$ implementação de ações nos diversos componentes da rede, disponibilização regional de serviços de saúde, aprimoramento dos mecanismos regulatórios e de comunicação entre os serviços.

Palavras-chave Hospitais, Regionalização, Assistência integral à saúde, Saúde pública 


\section{Introdução}

A estruturação das redes de saúde já era prevista desde a implementação do Sistema Único de Saúde (SUS), com propostas de condições para a construção de um sistema que permitisse o planejamento de suas estruturas e o atendimento das necessidades do indivíduo e da coletividade ${ }^{1}$. No entanto, na década de 1990, os próprios rumos do processo de descentralização tiraram a constituição de redes do foco central sobre o qual se pautaria a organização da assistência à saúde, evidenciando processos descoordenados de descentralização e regionalização ${ }^{2,3}$. Foi a partir dos anos 2000, com a divulgação das normas operacionais (NOAS) e, mais tardiamente, pelo Pacto pela Saúde, em 2006, que o planejamento territorial para a organização da atenção em saúde passou a ser valorizado com o intuito de elaborar políticas de saúde regionais e, consequentemente, estruturar as redes para enfrentar a dimensão territorial ${ }^{3}$.

Este processo por fortalecido a partir da Portaria 4.279/2010 e, neste contexto, o desenvolvimento da assistência à saúde, organizada em Redes de Atenção à Saúde (RAS), consistiu em uma proposta importante para a intercessão dos pontos de atenção, de forma dinâmica e complementar, apresentando-se como estratégia para a superação do modelo fragmentado de assistência à saúde ${ }^{1-4}$.

Em meio a estes debates surgem àqueles relacionados ao papel dos hospitais nestas RAS. Mesmo que na consolidação do SUS a proposta de reorientação do modelo assistencial fosse prioritária, com investimento na APS e tendências à desospitalização, muitas dificuldades foram encontradas devido à maior valorização dos cuidados hospitalares ${ }^{4}$. Manteve-se, ao longos dos anos, o modelo hospitalocêntrico detentor de determinantes históricos relacionados à forma como se buscou, ao longo dos anos, solucionar os problemas de saúde, com ênfase na doença $a^{5}$. E, como resultado, evidencia-se a alta demanda para assistência à saúde em nível hospitalar, já que um quarto das consultas ambulatoriais e $80 \%$ dos atendimentos de urgência e emergência ainda são realizados nestes estabelecimentos ${ }^{6}$.

Em 2014, os serviços de saúde com leitos para internação estavam distribuídos em aproximadamente 3,5 mil municípios, o que representava, aproximadamente, $63 \%$ do total de municípios brasileiros $^{7}$. Destes hospitais, $80 \%$ possuem pequeno e médio porte ${ }^{8}$, estando a maioria dos leitos sob a responsabilidade dos Hospitais Gerais
(HG), que consistem em estabelecimentos destinados a prestar atendimentos em especialidades básicas dispondo ou não de Serviço de Urgência e Emergência9. Acredita-se que o aumento da demanda por atenção hospitalar é muito evidenciada nos HG, fato que, somado à dificuldade de definição de papéis dos mesmos no sistema de saúde, reforça ainda mais os problemas de alinhamento deste serviço com os demais componentes das RAS e suas áreas prioritárias e, além disso, compromete os esforços desenvolvidos na gestão dos mesmos para sua plena atuação em redes $^{5}$. Este contexto relacionado aos HG contrapõe-se à realidade dos hospitais especializados, que possuem um conjunto de atividades de cuidados em saúde particulares e específicos, e que requerem fluxos previamente determinados e já direcionados para os usuários do sistema.

Partindo desta situação, as pesquisadoras buscaram conhecer, a partir da análise de outros estudos, suas contribuições a respeito da inserção dos HG no sistema de saúde, bem como os fatores que influenciam sua inserção nas RAS, considerando a importância da participação do sistema hospitalar brasileiro e sua relevância social e econômica para a operacionalização de um sistema resolutivo de redes. Desta forma, este estudo objetivou analisar a inserção dos hospitais gerais nas RAS e os fatores que condicionam o seu modo de participação nestas redes.

\section{Metodologia}

Para o desenvolvimento desta pesquisa, realizouse um scoping review que consiste em um método de mapeamento de literaturas relacionadas a temas de interesse e que reúne técnicas capazes de sintetizar os conhecimentos referentes a determinado tema em investigação, com amplitude e maior flexibilidade, não limitando o pesquisador a campos restritos de análise ${ }^{10}$.

O problema que impulsionou a busca bibliográfica por meio deste scoping review foi o interesse pela análise da inserção dos HG nas RAS e dos fatores que condicionam seu modo de participação nestas redes. Foram definidas como perguntas desta pesquisa: como se dá a inserção dos hospitais gerais nas RAS? Quais fatores condicionam seu modo de participação nestas redes?

Para a busca dos estudos utilizaram-se como critérios de inclusão: o período de publicação que se deu de janeiro de 2011 a junho de 2018, tendo em vista a publicação da Portaria no $4.279^{4}$; produções sobre o sistema de saúde brasileiro; a 
disponibilidade de estudos online e gratuitos; e o objeto de estudo. Como critérios de exclusão foram considerados livros ou capítulos de livro, dissertações, monografias e teses, documentos ministeriais e estudos do tipo revisão; estudos que envolvem hospitais privados que não atendem pelo sistema público de saúde e hospitais especializados.

A busca foi realizada no período de 01 a 28 de fevereiro de 2017 com atualização da pesquisa no mês de outubro de 2018, nas bases de dados Literatura Latino-Americana e do Caribe em Ciências da Saúde - Lilacs, United States National Library of Medicine at the National Institutes of Health - PubMed e na biblioteca eletrônica Scientific Eletronic Library Online - SciELO. Considerando o tema e o objeto estudado, optou-se por utilizar seguintes Descritores em Ciências da Saúde - DeCS: Hospitais, Hospitals, Regionalização, Regional health planning, Assistência Integral a Saúde, Comprehensive health care, Saúde Pública, Public health, e a expressão Rede de Atenção à Saúde (expressão), Health care networks, considerando sua importância para esta pesquisa e o fato de sua não existência no banco DeCS.

Os descritores citados foram dispostos em cruzamentos para a busca na base de dados, da seguinte forma: Hospitais AND Regionalização; Hospitais AND Saúde Pública; Hospitais AND Assistência Integral a Saúde; Hospitais AND Rede de Atenção à Saúde (palavra).

O processo de seleção dos estudos relevantes para esta pesquisa foi desenvolvido a partir da análise realizada por dois juízes, sendo eles os dois autores do presente estudo. Este processo "duplo" de análise iniciou-se a partir da leitura dos resumos, já que todos os estudos identificados na busca nas bases de dados foram inicialmente avaliados pelo autor 1, por meio da análise dos títulos. Neste processo, cada juiz tomou como base as questões norteadoras e os critérios de inclusão e exclusão previamente definidos.

Houve a seleção inicial de 1.323 referências na biblioteca eletrônica SciELO (680), nas bases de dados Lilacs (168) e no PubMed (367), sendo os mesmos submetidos à análise dos títulos. Ressalta-se que 10 dos estudos selecionados nesta primeira etapa foram encontrados em mais de uma base de dados ou obtidos em mais de um cruzamento, sendo os mesmo considerados apenas uma vez. A partir da análise dos títulos, selecionou-se 89 estudos que foram submetidos à análise dos resumos pelos dois juízes desta pesquisa.

A análise dos resumos por dois juízes gerou 80 concordâncias ( 28 inclusões e 52 exclusões) e cinco discordâncias. Os 28 estudos incluídos foram direcionados automaticamente para análise na íntegra e os cinco estudos que geraram discordância foram analisados pela dupla de juízes, utilizando-se a técnica de consenso na qual é verificado conjuntamente se os resumos respondiam à pergunta de pesquisa. A partir desta etapa, chegou-se à conclusão de que mais duas referências seriam inclusas, obtendo-se o total de 30 estudos que foram analisados na íntegra por ambos os juízes.

Na leitura dos trabalhos na íntegra houve concordância em todas as leituras, gerando a exclusão de mais 19 estudos. Desta forma, integraram a presente revisão 11 estudos que respondiam a pergunta desta pesquisa. Da análise das referências contidas nos artigos submetidos à leitura, foi possível identificar mais três estudos para compor este scoping review, totalizando 14 artigos (Figura 1).

A partir da análise dos estudos na íntegra, foi realizado o mapeamento dos dados obtidos, com a extração de informações relativas ao local e ano de publicação, título do artigo, autores, objetivos, metodologia, principais resultados e conclusões relacionadas à pergunta do estudo.

Dos estudos encontrados, a maioria $(35,8 \%)$ foi publicada no ano de 2015. Quanto ao local de estudo, $14,3 \%$ referiam-se a pesquisa de âmbito nacional, $64,3 \%$ foram realizadas na região sudeste, sendo as demais realizadas na região Nordeste $(7,1 \%)$ e Sul $(14,3)$. O Quadro 1 mostra, de forma resumida, a descrição dos trabalhos que fizeram parte do estudo.

A Figura 2 apresenta os percentuais de publicação segundo as áreas temáticas, demonstrando predominância das discussões referentes à integração e ao funcionamento da RAS $(28,6 \%)$ e ao perfil dos pacientes atendidos nos hospitais e Condições Sensíveis à Atenção Primária - CSAP $(28,6 \%)$.

Para a análise dos estudos levantados e mapeados, utilizou-se como referencial os Fundamentos das RAS propostos por Mendes ${ }^{11}$, apresentados e descritos no Quadro 2 e que foram considerados como categorias analíticas e apoiaram a análise das variáveis estudadas a partir dos estudos levantados. A partir desta organização, foi possível a criação de categorias empíricas de análise, sendo elas: "Hospital, regionalização e economia de escala"; "Fragilidade da organização da rede e hospitais como primeira escolha" e o "Hospital e evidências de (des)integração da RAS".

A Figura 3 apresenta as diversas relações obtidas entre os fundamentos das RAS proposto 


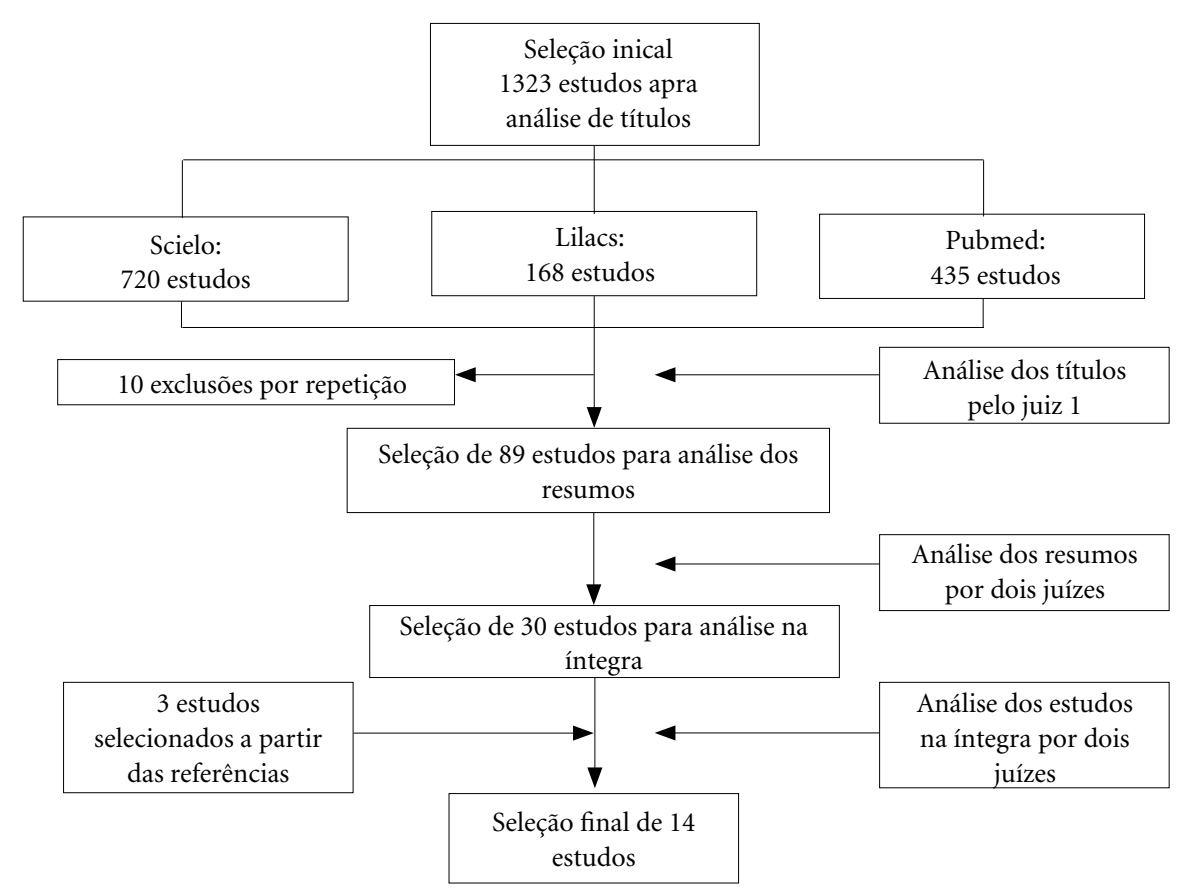

Figura 1. Fluxo demonstrativo das etapas percorridas na seleção dos estudos.

por Mendes ${ }^{11}$ (categorias analíticas), as variáveis analisadas e as categorias de análise criadas (categorias empíricas); e que permitirão subsidiar a discussão em cada categoria criada.

\section{Resultados}

A análise realizada neste estudo permitiu identificar alguns fatores que condicionam o modo como os serviços hospitalares estão inseridos nas RAS e se dispõe de acesso para a população. Identificou-se que a efetividade da atenção hospitalar está sujeita aos impactos da distribuição heterogênea destes estabelecimentos, bem como das fragilidades dos mecanismos de planejamento das regiões de saúde, da comunicação em rede e da integração entre os serviços, sejam eles no mesmo nível assistencial ou entre diferentes níveis de atenção. Esta situação leva à escassez da assistência hospitalar em algumas regiões ao mesmo tempo em que a população visualiza o hospital como porta de entrada do sistema, com distorção de seu papel como componente das RAS.

\section{Hospital, regionalização e economia de escala}

O processo de regionalização dos serviços de saúde tem sido alvo de discussões desde meados da década de 1990, com o lançamento de estratégias para a municipalização das ações na perspectiva de efetivação do princípio da integralidade e equidade no acesso aos serviços ${ }^{12}$. No entanto, à época pouco se efetivou nesse sentido sendo realmente colocado como ponto importante de organização do sistema de saúde a partir das NOAS 2001 e 2002 $2^{13,14}$; mais tardiamente pela divulgação do Pacto pela Saúde, em 2006, que visionou a integração dos sistemas municipais; e em 2010, por meio da Portaria Ministerial no 4.279, de dezembro de 2010, que estabeleceu as diretrizes para a organização da RAS no âmbito do SUS ${ }^{2,3,15}$. No ano seguinte, a publicação do Decreto 7.508 reforçou este processo ao regulamentar a Lei 8.080 de 1990 e dispor sobre o planejamento da saúde e da articulação interfederativa ${ }^{16}$.

A configuração da RAS foi proposta sob a ótica da relação entre a oferta de serviços e as necessidades dos usuários ${ }^{12,17}$. Operacionalmente, 
Quadro 1. Descrição dos estudos selecionados e o percentual obtido por ano de publicação, janeiro de 2011 a julho de 2018.

\begin{tabular}{|c|c|c|c|c|}
\hline Ano & Periódico & Local do estudo & Autores & Título do Artigo \\
\hline \multirow[t]{2}{*}{$\begin{array}{l}2011 \\
(14,3 \%)\end{array}$} & $\begin{array}{l}\text { Ciência e Saúde } \\
\text { Coletiva }\end{array}$ & $\begin{array}{l}\text { Estado de São } \\
\text { Paulo }\end{array}$ & $\begin{array}{l}\text { Venancio SI, Nascimento } \\
\text { PR, Rosa TE, Morais MLS, } \\
\text { Martins PN, Voloschko A } \\
\end{array}$ & $\begin{array}{l}\text { Referenciamento regional em saúde: } \\
\text { estudo comparado de cinco casos no } \\
\text { Estado de São Paulo, Brasil. }\end{array}$ \\
\hline & $\begin{array}{l}\text { Revista } \\
\text { Brasileira de } \\
\text { Saúde Materno } \\
\text { Infantil }\end{array}$ & $\begin{array}{l}\text { Município do } \\
\text { norte de Minas } \\
\text { Gerais }\end{array}$ & $\begin{array}{l}\text { Caldeira AP, Fernandes VBL, } \\
\text { Fonseca WP, Faria AA }\end{array}$ & $\begin{array}{l}\text { Internações pediátricas por condições } \\
\text { sensíveis à atenção primária em } \\
\text { Montes Claros, Minas Gerais, Brasil. }\end{array}$ \\
\hline $\begin{array}{l}2012 \\
(7,1 \%)\end{array}$ & $\begin{array}{l}\text { PhysisRevista de } \\
\text { Saúde Coletiva }\end{array}$ & $\begin{array}{l}\text { Rio de Janeiro/ } \\
\text { RJ }\end{array}$ & $\begin{array}{l}\text { Duarte JG, Gomes SC, Pinto } \\
\text { MT, Gomes MASM }\end{array}$ & $\begin{array}{l}\text { Perfil dos pacientes internados em } 199 \\
\text { serviços de pediatria no município do } \\
\text { Rio de Janeiro: mudamos? }\end{array}$ \\
\hline \multirow[t]{2}{*}{$\begin{array}{l}2013 \\
(14,3 \%)\end{array}$} & $\begin{array}{l}\text { PhysisRevista de } \\
\text { Saúde Coletiva }\end{array}$ & $\begin{array}{l}\text { Estado de } \\
\text { Pernambuco }\end{array}$ & $\begin{array}{l}\text { Dubeux LS, Freese E, } \\
\text { Felisberto E }\end{array}$ & $\begin{array}{l}\text { Acesso a Hospitais Regionais de } \\
\text { Urgência e Emergência: abordagem aos } \\
\text { usuários para avaliação do itinerário e } \\
\text { dos obstáculos aos serviços de saúde. }\end{array}$ \\
\hline & $\begin{array}{l}\text { Revista Gaúcha } \\
\text { de Enfermagem }\end{array}$ & \begin{tabular}{|l|} 
Municípios da \\
metade sul do \\
Rio Grande do \\
Sul \\
\end{tabular} & $\begin{array}{l}\text { Santos VCF, Kalsing A, Ruiz } \\
\text { ENF, Roese A, GerhardtTE }\end{array}$ & $\begin{array}{l}\text { Perfil das internações por doenças } \\
\text { crônicas não-transmissíveis sensíveis à } \\
\text { atenção primária em idosos da metade } \\
\text { sul do RS. }\end{array}$ \\
\hline $\begin{array}{l}2014 \\
(7,1 \%)\end{array}$ & $\begin{array}{l}\text { Revista da } \\
\text { Escola de } \\
\text { Enfermagem da } \\
\text { USP } \\
\end{array}$ & São Paulo/SP & $\begin{array}{l}\text { Rehem TCMSB, Ciosak SI, } \\
\text { EgryEY }\end{array}$ & $\begin{array}{l}\text { Internações Sensíveis à Atenção } \\
\text { Primária: usuários detectam falha no } \\
\text { acesso aos serviços }\end{array}$ \\
\hline \multirow[t]{5}{*}{$\begin{array}{l}2015 \\
(35,8 \%)\end{array}$} & Saúde Debate & $\begin{array}{l}\text { Estado de } \\
\text { Minas Gerais }\end{array}$ & $\begin{array}{l}\text { Lima DP, Leite MTS, } \\
\text { Caldeira AP }\end{array}$ & $\begin{array}{l}\text { Redes de Atenção à Saúde: a percepção } \\
\text { dos médicos trabalhando em serviços } \\
\text { de urgência }\end{array}$ \\
\hline & $\begin{array}{l}\text { Saúde e } \\
\text { Sociedade }\end{array}$ & $\begin{array}{l}\text { Estado de } \\
\text { Minas Gerais }\end{array}$ & $\begin{array}{l}\text { Torres SFS, Belisário AS, } \\
\text { Melo EM }\end{array}$ & $\begin{array}{l}\text { A rede de urgência e emergência da } \\
\text { macrorregião norte de Minas Gerais: } \\
\text { um estudo de caso }\end{array}$ \\
\hline & $\begin{array}{l}\text { Rev Saúde } \\
\text { Pública }\end{array}$ & $\begin{array}{l}\text { Estado de São } \\
\text { Paulo }\end{array}$ & \begin{tabular}{|l|} 
Pazin-Filho A, Almeida E, \\
Cirilo LP, Lourençato FM, \\
Baptista LM, Pintyá JP, \\
Capeli RD, Silva SMPF, Wolf \\
CM, Dinardi MM, Scarpelini \\
S, Damasceno MC \\
\end{tabular} & $\begin{array}{l}\text { Impacto de leitos de longa } \\
\text { permanência no desempenho de } \\
\text { hospital terciário em emergências. }\end{array}$ \\
\hline & $\begin{array}{l}\text { Revista Saúde e } \\
\text { Sociedade }\end{array}$ & $\begin{array}{l}\text { Grande Vitória/ } \\
\text { ES }\end{array}$ & Barreto Junior IF & $\begin{array}{l}\text { Regionalization of public hospital care } \\
\text { in the metropolitan area of Vitória, } \\
\text { Espírito Santo, Brazil }\end{array}$ \\
\hline & $\begin{array}{l}\text { Rev Saúde } \\
\text { Pública }\end{array}$ & São Paulo/SP & $\begin{array}{l}\text { Ramos MCA, Cruz LP, } \\
\text { Kishima VC, Pollara WM, } \\
\text { Lira ACO, Couttolenc BF } \\
\end{array}$ & $\begin{array}{l}\text { Avaliação de desempenho de hospitais } \\
\text { que prestam atendimento pelo sistema } \\
\text { público de saúde, Brasil. }\end{array}$ \\
\hline $\begin{array}{l}2016 \\
(7,1 \%)\end{array}$ & $\begin{array}{l}\text { Saúde em } \\
\text { Debate }\end{array}$ & Brasil & $\begin{array}{l}\text { Pessôa LR, Santos IS, } \\
\text { Machado JP, Martins ACM, } \\
\text { Lima CRA }\end{array}$ & $\begin{array}{l}\text { Realocar a oferta do SUS para atender } \\
\text { problemas do futuro: o caso do trauma } \\
\text { no Brasil }\end{array}$ \\
\hline $\begin{array}{l}2017 \\
(7,1 \%)\end{array}$ & $\begin{array}{l}\text { International } \\
\text { Journal for } \\
\text { Equity in Health }\end{array}$ & Brasil & $\begin{array}{l}\text { Rocha TAH, Silva NC, } \\
\text { Amaral PV, Barbosa ACQ, } \\
\text { Rocha JVM, Alvares V, } \\
\text { Almeida DG, Thumé E, } \\
\text { Thomaz EBAF, Queiroz RCS, } \\
\text { Souza MR, Lein A, Lopes } \\
\text { DP, Staton CA, Vissoci JRN, } \\
\text { Facchini LA }\end{array}$ & $\begin{array}{l}\text { Addressing geographic access barriers } \\
\text { to } \\
\text { Emergency care services: a national } \\
\text { Ecologic study of hospitals in Brazil. }\end{array}$ \\
\hline $\begin{array}{l}2018 \\
(7,1 \%)\end{array}$ & $\begin{array}{l}\text { Cadernos de } \\
\text { Saúde Pública }\end{array}$ & $\begin{array}{l}\text { Estado de Santa } \\
\text { Catarina }\end{array}$ & $\begin{array}{l}\text { Cirino S, Gonçalves LA, } \\
\text { Gonçalves MB, Cursi ES }\end{array}$ & $\begin{array}{l}\text { A nonlinear model for localization } \\
\text { of hospital services as an indicator of } \\
\text { accessibility }\end{array}$ \\
\hline
\end{tabular}




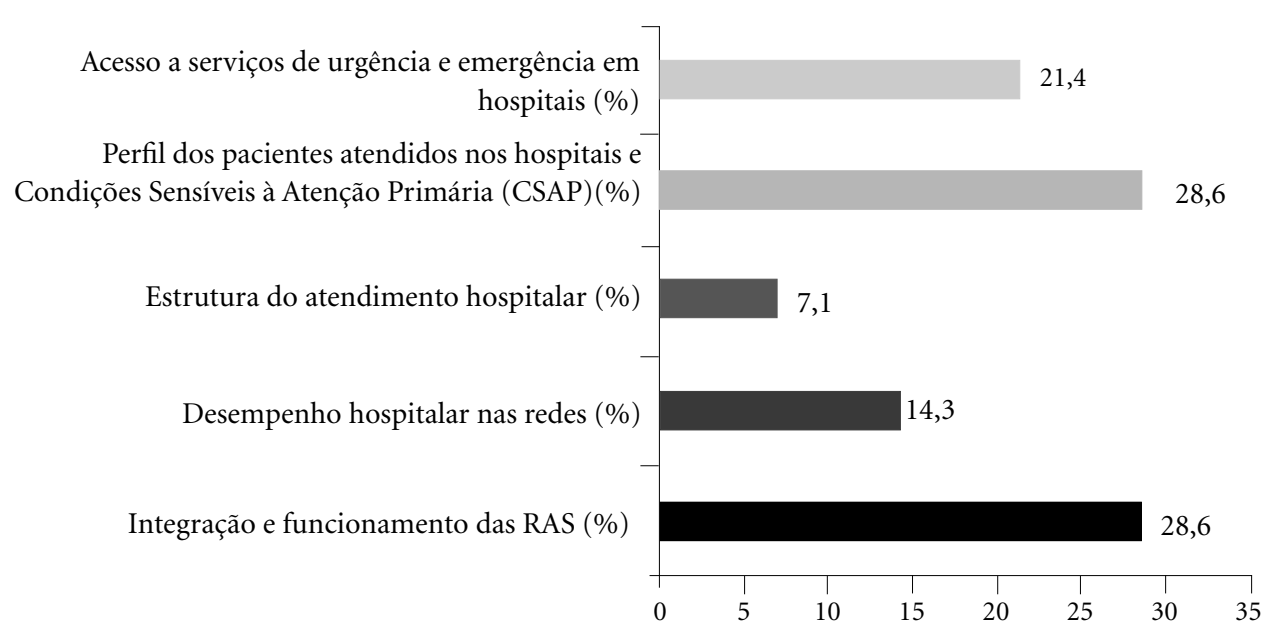

Figura 2. Distribuição dos estudos analisados por área temática, janeiro de 2011 a junho de 2018.

Quadro 2. Fundamentos das Redes de Atenção à Saúde propostos por Mendes ${ }^{11}$ e seus conceitos.

\begin{tabular}{|l|}
\hline \multicolumn{1}{|c|}{ Economia de Escala, qualidade e acesso } \\
\hline $\begin{array}{l}\text { Organização das RAS de forma que serviços com menor economia de escala devem estar distribuídos de } \\
\text { maneira mais dispersa, com recursos suficientes e para o qual a distância é fator fundamental de acessibilidade. } \\
\text { Já os serviços com maior economia de escala devem estar alocados de maneira concentrada, uma vez contendo } \\
\text { recursos mais escassos e para o qual a distância tem menor impacto sobre o acesso. }\end{array}$ \\
\hline \multicolumn{1}{c|}{ Territórios Sanitários } \\
\hline $\begin{array}{l}\text { Fundamenta a articulação do território com as RAS, considerando a necessidade de se planejar a assistência } \\
\text { com base nas características do território e da população. }\end{array}$ \\
\hline \multicolumn{1}{|c|}{ Integração horizontal e vertical } \\
\hline $\begin{array}{l}\text { Integração horizontal acontece entre unidades de produção semelhantes, visando o adensamento da produção } \\
\text { e ganhos em escala enquanto a Integração vertical se dá entre unidades de produção. }\end{array}$ \\
\hline $\begin{array}{l}\text { Agrupamento de substituição } \\
\text { reposta às necessidades da população e dos recursos disponíveis. }\end{array}$ \\
\hline \multicolumn{1}{|c|}{ Níveis de atenção } \\
\hline $\begin{array}{l}\text { Arranjos produtivos estruturados conforme as diferentes densidades tecnológicas singulares desde a APS até a } \\
\text { atenção secundária e terciária. }\end{array}$ \\
Fonte: Mendes ${ }^{11}$.
\end{tabular}

este processo está respaldado pelos ideais de articulação entre serviços, delimitação de territórios e planejamento da assistência à saúde, segundo seu perfil epidemiológico e sanitário, tendo a APS como ordenadora do cuidado e direcionadora do usuário para os demais níveis de complexida$\mathrm{de}^{15,18}$.

A lógica de organização dos serviços em redes deveria responder aos determinantes da qualidade e efetividade por meio da disposição de estabelecimentos segundo Economia de Escala
(EE), com a concentração de serviços com recursos mais escassos e para os quais a distância gerará menor impacto sobre o acesso, contando com um sistema regulatório efetivo com ordenação e coordenação da APS $^{11}$. Nesta perspectiva, a complexidade hospitalar alocada regionalmente justifica a possibilidade de deslocamento de usuários na rede e a necessidade de efetiva comunicação e articulação entre os serviços, extrapolando os limites de referência do município. Assim, a maneira como a EE está disponibilizada no terri- 


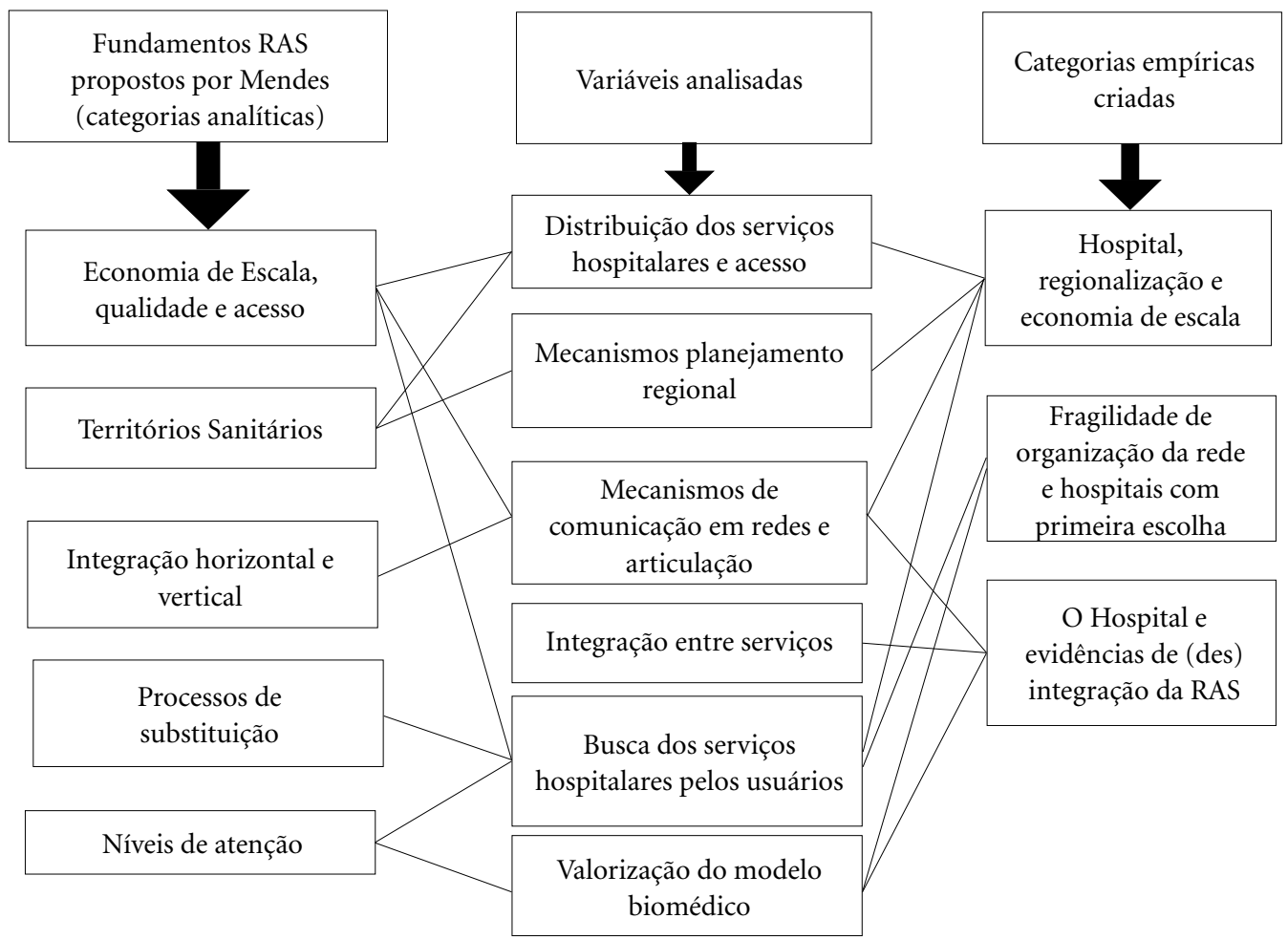

Figura 3. Esquema de relações entre os fundamentos das RAS proposto por Mendes, as variáveis analisadas e as categorias de análise criadas.

tório e a efetivação dos mecanismos regulatórios, partindo de um qualificado planejamento regional, determinam as facilidades e as dificuldades de acesso do usuário aos serviços de saúde ${ }^{19}$.

Os estudos mostram que, apesar de esforços para que o arcabouço legislativo referente às RAS seja colocado em prática, o processo de regionalização dos serviços de saúde não foi efetivamente instituído e sofre vieses que impactam no modo como os componentes da RAS se apresentam enquanto estabelecimentos de assistência à saúde ${ }^{20}$.

A assistência hospitalar, componente importante na oferta de escala às regiões de saúde, muitas vezes apresenta-se distribuída inadequadamente condicionando sua inserção na rede de maneira desordenada, desarticulada dos demais serviços e não atendendo as demandas da população. Rocha et al..$^{21}$, ao pesquisar sobre as barreiras de acesso aos serviços de Urgência e Emergência, mostrou que as Regiões Norte e
Centro-Oeste sofrem com escassez de assistência hospitalar, com áreas cuja distribuição de leitos por habitante se aproxima de zero. Ao passo em que isto acontece, existe uma grande concentração de hospitais pequenos e pouco resolutivos no território nacional, sendo estes a maioria dos estabelecimentos hospitalares brasileiros.

Considerando a distribuição dos serviços hospitalares e o acesso como variáveis analisadas neste estudo, é pertinente refletir sobre o fato de que $55,9 \%$ dos hospitais pequenos estão localizados a menos de 60 quilômetros de distância dos serviços hospitalares de maior complexidade. Entende-se, desta forma, que os demais estabelecimentos deste porte se encontram em regiões distantes de serviços de maior aporte tecnológico, dificultando o acesso do usuário. Soma-se, ainda, o fato de que é escassa a disponibilidade de centros de maior complexidade em algumas regiões, com apenas $7,76 \%$ dos municípios bra- 
sileiros contemplados, visualizando-se melhor acessibilidade nos estados de Paraná, Goiás, Minas Gerais, Bahia, Piauí, Rio Grande do Norte, Ceará e Pernambuco ${ }^{20}$.

Sob a ótica da EE, existem muitas discussões a respeito da efetividade dos HPP e da manutenção dos mesmos. Estes estabelecimentos apresentam baixas taxas de ocupação e má relação custo-efetividade; falta de estrutura, de recursos humanos e dificuldades na organização do trabalho, muitas vezes não conseguindo se colocar com um papel definido na rede de assistência. A maioria destes estabelecimentos cumpre um papel semelhante ao da APS e, ao passo em que não alcança economias ótimas de escala, desenvolve a incapacidade de prestar cuidados, impondo ao usuário a necessidade de deslocamento ${ }^{5,21}$.

A inadequação na distribuição dos serviços hospitalares, quantitativa ou qualitativa, leva a existência de vazios assistenciais em algumas regiões, ocasionando a "evasão" de usuários, caracterizada pelo deslocamento de moradores para outras regiões em busca de assistência. Esta consiste em uma informação gerencial importante para a análise da efetividade das regiões de saúde, bem como para a distribuição dos serviços hospitalares e da articulação em rede ${ }^{17}$.

Pesquisa realizada sobre as práticas gestoras de referenciamento regional no Estado de São Paulo mostrou que a assistência hospitalar é mais presente em algumas regiões em detrimento de outras, sendo que a região metropolitana de São Paulo alberga um grande centro tecnológico em saúde e atrai usuários de outras regiões de saú$\mathrm{de}^{18}$. Da mesma maneira ocorre na Região Metropolitana da Grande Vitória (ES), cuja capital possui $18,7 \%$ da população desta região, no entanto, absorve mais de 53\% das internações dos municípios, evidenciando a instalação da maior oferta de leitos hospitalares na capital ${ }^{17}$.

Torres et al..$^{22}$, mostraram que a má distribuição dos hospitais em algumas microrregiões acarreta no não cumprimento de pactuações e sobrecarrega a macrorregião no atendimento hospitalar para a média e a alta complexidade. Campinas, mesmo constituindo-se em um grande centro de serviços de saúde de média e alta complexidade, com tecnologias disponíveis de maneira concentrada, revela-se como insuficiente para o atendimento à demanda por serviços hospitalares, uma vez que seu grupo de usuários é constituído pela população residente na região somada àquela de outras regiões e que é atraída pelo alto aporte tecnológico ${ }^{18}$. Ainda, estudo realizado em HG do Rio de Janeiro demonstrou que $56 \%$ dos usuários atendidos procediam desta capital, enquanto, $44 \%$ eram provenientes de outros municípios, o que demonstra a tendência de deslocamento da população em direção aos serviços hospitalares dos grandes centros ${ }^{23}$.

Se por um lado a concentração de alguns serviços, sob a lógica da EE, propõe uma organização do acesso, por outro, a depender da maneira como acontece o planejamento regional e a implementação dos mecanismos regulatórios, a concentração de tecnologias representa obstáculo no acesso à atenção em saúde. Isso acontece especialmente em regiões onde ocorre a escassez de serviços de menor densidade tecnológica ou que se apresentam pouco integrados com os demais componentes da rede ${ }^{24}$. Do ponto de vista do usuário, alguns fatores são considerados como dificultadores do acesso à assistência hospitalar: a distância do hospital em relação às suas residências; a necessidade de pagamento do transporte para chegar ao hospital e a dependência de transporte de conhecidos ${ }^{15}$.

Cirino et al..$^{25}$ trazem uma reflexão a respeito da distribuição dos serviços de saúde e a correlação com os princípios de eficiência e equidade. Quando o sistema busca a otimização da eficiência, ele almeja que grande parte dos usuários seja provido por um determinado orçamento público, maximizando a distribuição dos serviços em determinado espaço geográfico. Ao buscar maior equidade, lança mão de mecanismos para distribuir os serviços de forma que os usuários despendam esforços equivalentes às suas necessidades, maximizando o efeito da assistência na população. Neste sentido, o conceito de eficiência equivale ao de escala, evidenciando que a existência de serviços em determinada região geográfica por si só não confere à rede o cumprimento do princípio de equidade.

As evidências mostram que, para um efetivo funcionamento do sistema hospitalar, partindo da disponibilidade racional nos diferentes territórios e, então, com a definição de seu papel nas diversas regiões de saúde, é imperativo que gestores busquem o planejamento das ações e mecanismos de estruturação de um sistema funcional pautados em estratégias efetivas e pactuações que extrapolem os limites municipais e buscando o aperfeiçoamento dos sistemas regulatórios e de comunicação interregional ${ }^{15,18}$.

Ainda, partindo do pressuposto da inefetividade dos HPP, são necessárias muitas discussões sobre a redefinição de seu papel e de suas práticas. Já existem políticas ministeriais de incentivo ao fechamento de leitos nestas unidades e aber- 
tura para atendimentos ambulatoriais, com práticas de referência e contra referência em rede ${ }^{6}$. Paralelamente a isso, há quem propõe o fechamento dos mesmos, a exemplo do Banco Mundial que, em relatório de análise da eficiência e equidade do gasto público no Brasil, sugere a racionalização da rede hospitalar, visando o alcance de melhor equilíbrio entre a escala e o acesso ${ }^{26}$. Este posicionamento pode levar ao agravamento do vazio assistencial em algumas regiões de saúde e não considera a possibilidade de sua restruturação, como propõem Pazin-Filho et al. ${ }^{27}$, ao atribuir aos HPP o atendimento a casos crônicos de alta dependência e baixa complexidade, com redução na ocupação de leitos de unidades maiores e disponibilização de espaço em áreas estranguladas do sistema como aquelas voltadas a cuidados intensivos.

\section{Fragilidade de organização da rede e hospitais com primeira escolha}

Os resultados do presente estudo apontaram para fragilidades da organização do sistema de saúde em redes como importante fator de determinação dos hospitais como primeira escolha do usuário mediante suas necessidades de cuidados à saúde.

Apesar de se esperar, a partir das ações de reforma originadas nas ultimas décadas do século XX, um processo de organização de um modelo de atenção à saúde, pautado na concepção de um sistema integrado de serviços que interligue ações de prevenção, promoção e reabilitação com a atenção curativa ${ }^{28}$, o que se observa, em diversas realidades é a desconfiguração da rede de atenção, com a distorção nos papéis de cada nível.

Especialmente, a APS sofre importantes impactos das dificuldades estruturais e operacionais que comprometem seu papel de componente detentor de ferramentas estratégicas para a reorganização do sistema e de articulador com os demais componentes da RAS ${ }^{18,29}$. Sem condições materiais, tecnológicas e organizacionais para seu pleno funcionamento ${ }^{15,29}$, estudos apontaram que, segundo usuários e profissionais de saúde, a atenção primária apresenta resolutividade diminuída ${ }^{21}$, dificultando a disponibilidade de seus serviços aos usuários, bem como o alcance dos serviços de média e alta complexidade a partir de um fluxo formal de acesso ${ }^{25}$, visto que muitas vezes não possui a capacidade operacional para exercer seu papel de centro comunicador entre os pontos que compõem as complexas redes de cuidado $^{24,29}$.
Na medida em que estes pontos interferem no pleno funcionamento da rede de serviço e criam obstáculos aos usuários na busca do atendimento de suas necessidades de saúde, criam-se fluxos informais de acesso aos serviços, revelando um potencial perfil atrativo dos serviços hospitalares, determinado pela oferta de especialidades e leitos, perfil de atendimento, reputação, bem como disponibilidade de elementos tecnológicos, apropriados para o tratamento da "doença" (medicalização da saúde) $)^{1,3,11,25}$.

Faz-se necessário destacar, neste contexto, que mesmo com todas as mudanças nos sistemas de saúde com reorientação das políticas a partir da crítica ao modelo centrado no hospital e, ainda, com as discussões sobre o papel da atenção hospitalar, esta segue mantendo um papel de destaque na prestação de assistência à saúde. Isso se dá tanto do ponto de vista técnico-assistencial com concentração de saberes e tecnologias, quanto a partir da ótica da mídia e da população que visualizam uma representação simbólica de um local privilegiado e verdadeiramente expressivo no que se refere a um serviço de saúde ${ }^{28}$.

Essa situação não é exclusiva do Brasil. Estudo que analisa as estratégias desenvolvidas por Brasil e Espanha para integrar Atenção Primária à Saúde e Especializada, descreve que na Espanha "apesar do amplo acesso à APS, cujas unidades funcionam em dois turnos, foi relatado [...] a existência de certa cultura, que considera a atenção hospitalar como de melhor qualidade", o que faz com que a população busque o atendimento diretamente na rede de urgência ${ }^{30}$.

Como fator de incremento à situação exposta, os profissionais de saúde exercem um papel de destaque na valorização da atenção hospitalar e seus instrumentos em detrimento de outros serviços, uma vez que enxergam e disseminam a ideia de possibilidades de uma atenção mais resolutiva, ao passo em que apontam para dificuldades de outros pontos da rede de serviços na disponibilização de recursos e revelam carência de conhecimentos sobre os percursos que o usuário pode percorrer no sistema. Desta maneira, evidencia-se fortemente a predominância da formação médica pautada no modelo especializado, colocando uma real urgência de análise e melhorias na formação acadêmica, de modo que permita a valorização do atendimento no âmbito da prevenção e promoção da saúde, com potencial garantia de efetividade da atenção em saúde e maiores possibilidades de reorganização dos servicos ${ }^{1}$.

A alta incidência de atendimentos hospitalares a usuários com Condições Sensíveis a Aten- 
ção Primária (CSAP) também ganhou destaque nesta seção, como consequência de inadequações da organização da rede e da baixa efetividade de ações de prevenção e promoção da saúde em algumas regiões. Em Montes Claros (MG), estudo mostrou que $41,4 \%$ das internações infantis decorreram de CSAP, sendo que 77,5\% destas crianças já eram acompanhadas por equipes do nível de atenção primário ${ }^{24}$. Pesquisa realizada com internações hospitalares no Rio Grande do Sul, de 2000 a 2010 , mostrou que $76,6 \%$ das internações por CSAP são originadas de Doenças Crônicas Não-Transmissíveis (DCNT) $)^{31}$.

As dificuldades apresentadas na organização do sistema de saúde em rede levam a uma situação de desencontro dos perfis epidemiológicos com o sistema de saúde, em que coexiste a predominância das condições crônicas e um sistema de atenção à saúde direcionada a responder aos eventos agudos e "agudizações" de condições crônicas de maneira reativa e fragmentada ${ }^{19}$, além do incremento na demanda para os hospitais gerais $^{15,22}$. Este representa um fator determinante na dificuldade de definição de um papel para a atenção hospitalar não especializada, uma vez que este perde seu perfil de componente articulado com a rede, na medida em que presta assistência a casos passíveis de atendimento em outros níveis e não se insere num processo comunicativo com a mesma ${ }^{24}$.

Como consequência da realidade exposta nesta seção, a alta e crescente demanda para a atenção hospitalar gera um quadro real de superlotação dos estabelecimentos, com redução na oferta de leitos disponíveis para atendimento dos usuários da RAS, além de desencadear sobrecarga aos trabalhadores e potencial declínio na qualidade do atendimento. Como consequência deste cenário, tem-se o descontentamento da população usuária e de reforço da ideia de que faltam hospitais no país ${ }^{1,3,15}$. Ainda, do ponto de vista econômico, esta alta e crescente demanda para a atenção hospitalar reflete um importante incremento nos gastos públicos em saúde, já que os gastos médicos hospitalares representam cerca de metade de todos os gastos nacionais em saúde, sendo um importante fortalecedor da preocupação já existente na década de 1970, com a necessidade de racionalização econômica e da oferta, bem como de modernização dos processos de gestão ${ }^{28}$.

\section{O Hospital e evidências de (des)integração da RAS}

O acesso, já discutido na sessão anterior, não se restringe apenas à procura e entrada do usuário no serviço de saúde, mas como isso ocorre. Envolve os processos de cuidado, com o alcance de suas etapas complementares e os resultados do trabalho desenvolvido em cada uma delas. Mediante este raciocínio, deve-se considerar a análise dos fluxos para a obtenção de assistência e por meio deles atentar-se para os processos de referência e contra referência inerentes que permitam assegurar os princípios da integralidade do cuidado e da universalidade da atenção em saú$\mathrm{de}^{12,15}$. A maneira como esta interlocução entre os níveis de atenção acontece, e sua efetividade, impactam significativamente na forma como os HG estão inseridos nas diversas RAS e as características do atendimento por eles prestados.

Entendem-se fluxos de referência e contra referência como um processo de encaminhamento dos pacientes, de maneira mútua, entre os diferentes níveis de complexidade dos serviços ${ }^{32}$. Este conceito traz consigo a ideia de integração da rede assistencial em saúde, cuja construção se baseia na conexão, comunicação, coerência e relação entre os serviços componentes da rede de cuidados ao usuário. Na medida em que se produz um cuidado com integração adequada entre os serviços, permite-se desenvolver um cuidado "completo" e "inteiro".

Para Mendes ${ }^{11}$, a integração pode ser aplicada de duas formas, sendo a horizontal aquela que permite o desenvolvimento do processo de cuidado por meio da fusão de serviços ou por aliança estratégica obtida com complementariedade em rede; e a vertical, que submete todos os pontos de atenção, de apoio e de logística a uma mesma gestão, com a interlocução obtida por meio de comunicação efetiva, gerando um valor na cadeia de produção de cuidado.

Mediante a falta de integração entre os componentes de uma RAS, visualiza-se a fragmentação do cuidado em saúde, exemplificada por Mendes ${ }^{11}$, ao pontuar situações referentes ao cuidado com doenças crônicas, em especial para os casos de hipertensão arterial no Brasil. Este agravo à saúde mantém-se como uma das doenças crônicas submetidas ao cuidado fragmentado e voltado para as condições agudas reativas à ocorrência.

Um grupo de médicos de unidades de urgência mineiras, participantes de pesquisa já citada neste estudo, pontuaram esta situação, eviden- 
ciada pela desconexão entre os componentes das RAS quando da inexistência de fluxos de referência e contra referência entre a unidade hospitalar da qual faziam parte e os demais níveis de atenção. Manifestaram também desconhecer a existência de comunicação efetiva entre gestores dos diversos níveis assistenciais e atribuíam isso como um fator determinante para as falhas no exercício da governança pelo Estado, uma vez que esta consiste em arena de articulação e pactuação entre os atores sociais e políticos ${ }^{1}$. Este fato agrava os problemas relativos à superlotação das unidades hospitalares, haja vista a impossibilidade que estes serviços enfrentam nas tentativas de encaminhamento dos usuários aos demais níveis de atenção. Ainda, esta situação leva ao uso inadequado de leitos e, consequentemente, pode gerar a ideia de ineficiência da média e alta complexidade hospitalar na rede de atenção à saúde.

Campinas, como uma sede regional que dispõe de grande oferta de tecnologia, só comparável à da RMGSP, porém com uma população bem inferior, não dispõe de mecanismos eficientes de regulação, de modo que demandas desnecessárias e externas à região sobrecarreguem os serviços e impeçam o atendimento das necessidades regionais. Esta região reconhece sua falta de governabilidade financeira e de instrumentos de identificação da necessidade e do encaminhamento da demanda, além de se constituir em uma arena de interesses de mercado. Tais circunstâncias dificultam o desenvolvimento de uma articulação permanente entre os gestores com o objetivo de prestar uma assistência voltada diretamente para as necessidades da população e, consequentemente, para a melhoria do desempenho do sistema ${ }^{18}$.

$\mathrm{Na}$ regional de Mogi das Cruzes, que possui os menores índices de hospitais, leitos de UTI e cobertura de ESF, detectou-se um grande esforço da gestão, com a criação de mecanismos de participação, de escuta populacional, de formação e negociação. No entanto, a complexidade metropolitana e a proximidade de São Paulo reduzem as possibilidades criadas pelo esforço realizado, somado à incapacidade regional de produzir objetivos comuns entre os gestores que, perseguindo objetivos locais, agem de maneira desintegra$\mathrm{da}^{18}$.

Uma das soluções para esta problemática está no efetivo fortalecimento regional das RAS, pautado na implementação de um sistema de governança de cunho analítico e investigativo sobre as condições de saúde, permissivo da troca de informações entre os componentes da rede e da transformação nos processos de gestão e de construção de políticas de saúde ${ }^{1,33}$.

Esta interlocução necessária entre os diferentes atores pôde ser observada em pesquisa realizada na Rede de Urgência e Emergência (RUE) de Minas Gerais, cujo estudo do seu Comitê Gestor permitiu evidenciar um processo de encontro e comunicação entre os diversos membros representantes dos componentes da rede (gestores, profissionais de saúde, prestadores de serviço e outros), que permeou a sensibilização e a divulgação da RAS até a realização de um diagnóstico, definição e tipologia dos pontos hospitalares, sendo uma aposta destes gestores para acertos no que se refere ao funcionamento desta RUE ${ }^{22}$.

\section{Conclusões}

Este estudo apresentou alguns fatores inerentes ao sistema de saúde atual que condicionam a maneira como os Hospitais atuam nas RAS, tendo destaque para a inadequada distribuição destes serviços e de seus níveis de escala nos territórios; a presença de um número considerável de HPP que, sem um papel definido, muitas vezes não atendem plenamente as demandas da população; as fragilidades da organização dos serviços em rede, com pouca articulação entre os pontos de atenção; e, ainda, a hegemonia do modelo biomédico.

A inadequada distribuição dos HG no território brasileiro, como resultado de planejamentos pautados na oferta e não na demanda definida com base no perfil da população, determina a possibilidade de desarticulação deste componente com o sistema e consequentemente sua má representatividade, imprimindo a imagem de ineficácia dos serviços hospitalares. A existência de estabelecimento hospitalar por si só, destacando-se aqui os HPP, não determina a qualidade na assistência deste nível de atenção, uma vez que não é garantida a disponibilidade dos recursos necessários, podendo inexistir sistemas efetivos de comunicação e integração entre os serviços para o pleno acesso à saúde.

A melhor qualificação, inserção e desenvolvimento HG nas RAS dependerá do enfrentamento, por parte dos gestores, de desafios relacionados ao planejamento e à implementação de ações nos diversos componentes da RAS, bem como na disponibilização regional dos mesmos, como fator determinante de melhores condições de acesso do usuário e de resolutividade dos problemas de saúde em cada nível de atenção do Sistema. 
É inerente a este processo, a potencialização da negociação e coordenação do sistema em um determinado território, ou seja, do efetivo uso de mecanismos de governança regional, o que implicaria na necessidade de redefinição dos papéis dos agentes do sistema de saúde e da política na efetivação dos sistemas de regulação do acesso, formalizada por meio de Contratos de Gestão entre diversos entes, bem como dos planos de regionalização, permitindo melhorar a eficiência dos investimentos e a eficácia dos serviços.

A definição de papéis do HG, quando inexistente, dependerá do perfil populacional, da existência de outros serviços na região, bem como da capacidade de articulação entre os atores envolvidos, sendo possível assumir diversas interfaces assistenciais a depender da região em que se insere e de sua demanda.

\section{Colaboradores}

FG Borsato e BG Carvalho contribuíram de maneira semelhante na construção deste artigo, especialmente na aplicação da metodologia descrita no mesmo, quando da análise dos estudos levantados por dois juízes (pesquisadores). 


\section{Referências}

1. Lima DP, Leite MTS, Caldeira AP. Redes de Atenção à Saúde: a percepção dos médicos trabalhando em serviços de urgência. Saúde Debate 2015; 39(104):65-75.

2. Kuschinir R, Chorny AH. Redes de atenção à saúde: contextualizando o debate. Cien Saude Colet 2010; 15(5):2307-2316.

3. Viana ALD, Bousquat A, Melo GA, Negri Filho A, Medina MG. Regionalização e Redes de Saúde. Cien Saude Colet 2018; 23(6):1791-1798.

4. Brasil. Portaria no 4.279, de 30 de dezembro de 2010. Estabelece diretrizes para a organização da Rede de Atenção à Saúde no âmbito do Sistema Único de Saúde (SUS). Diário Oficial da União 2010; $30 \mathrm{dez}$.

5. Rosa T. O papel do hospital na Rede de Atenção a Saúde. Consensus 2014; 4(11):13-23.

6. Ramos MCA, Cruz LP, Kishima VC, Pollara WM, Lira ACO, Couttolenc BF. Avaliação de desempenho de hospitais que prestam atendimento pelo sistema público de saúde, Brasil. Rev Saude Publica 2015; 49:43.

7. IBGE divulga as estimativas populacionais dos municípios em 2015. Agência IBGE notícias; 2015 Ago 28.

8. Pessoa LR, Santos IS, Machado JP, Martins ACM, Lima CRA. Realocar a oferta do SUS para atender problemas do futuro: o caso do trauma no Brasil. Saúde Debate 2016; 40(110):9-19.

9. Brasil. Ministério da Saúde. Cadastro Nacional de Serviços de Saúde (CNES) [Internet]. Brasília: MS. [acessado 2016 Nov 17]. Disponível em: http://tabnet. datasus.gov.br/cgi/cnes/tipo_estabelecimento.htm

10. Arksey H, O'Malley L. Scoping studies: towards a methodological framework. Int J Soc Res Methodol 2005; 8(1):19-32.

11. Mendes EV. As redes de atenção à saúde. Brasília: Organização Pan-Americana da Saúde; 2011.

12. Travassos C, Castro MSM. Determinantes e desigualdades sociais no acesso e na utilização dos serviços de saúde. In: Giovanella L, Escorel S, Lobato, LVC, Noronha JC, Carvalho AI, organizadores. Políticas e sistemas de saúde no Brasil. Rio de Janeiro: Fiocruz; 2012. p. 183-206.

13. Brasil. Portaria no 95, de 26 de janeiro de 2001. Norma Operacional da Assistência à Saúde. NOAS-SUS 01/2001. Diário Oficial da União 2001; 26 jan.

14. Brasil. Portaria $n^{\circ} 373$, de 27 de fevereiro de 2002. Norma Operacional da Assistência à Saúde. NOASSUS 01/2002. Diário Oficial da União 2002; 27 fev.

15. Dubeux LS, Freese E, Felisberto E. Acesso a hospitais regionais de urgência e emergência: abordagem aos usuários para avaliação do itinerário e dos obstáculos aos serviços de saúde. Physis [serial on the Internet]. 2013 Jun [cited 2017 may 5]; 23(2): [about 21 p.]. Available from: http://www.scielo.br/pdf/physis/ v23n2/v23n2a03.pdf

16. Brasil. Decreto 7.508 de 28 de junho de 2011. Regulamenta a Lei $\mathrm{n}^{\circ} 8.080$, de 19 de setembro de 1990, para dispor sobre a organização do Sistema Único de Saúde - SUS, o planejamento da saúde, a assistência à saúde e a articulação interfederativa, e dá outras providências. Diário Oficial da União 2011; 28 jun.
17. Barreto Junior IF. Regionalization of public hospital care in the metropolitan área of Vitória, Espírito Santo, Brazil. Saude soc. [serial on the Internet]. 2015 Jun [cited 2017 Sep 1];24(2):[about 10 p.]. Available from: http://www.scielo.br/pdf/sausoc/v24n2/en_01041290-sausoc-24-02-00461.pdf

18. Venâncio SI, Nascimento PR, Rosa TE, Morais MLS, Martins PN, Voloschko A. Referenciamento regional em saúde: estudo comparado de cinco casos no Estado de São Paulo, Brasil. Cien Saude Colet 2011; 16(9):3951-3964.

19. Coelho MF, Chaves LDP, Anselmi ML, Hayashida M, Santos CB. Análise dos aspectos organizacionais de um serviço de urgências clínicas: estudo em um hospital geral do município de Ribeirão Preto, SP, Brasil. Rev. Latino-Am. Enfermagem [serial on the Internet]. 2010 Jul-Ago [cited 2018 Jun 22];18(4):[about 9 p.]. Available from: www.eerp.usp.br/rlae

20. Almeida PF, Santos AM, Santos VP, Silveira Filho RM. Integração assistencial em região de saúde: paradoxo entre necessidades regionais e interesses locais. Rev Saude Soc [serial on the Internet]. 2016 Abr-Jun [cited 2018 oct 20];25(2):[about 15 p.]. Available from: https:// doi.org/10.1590/S0104-12902016153295

21. Rocha TAH, Silva NC, Amaral PV, Barbosa ACQ, Rocha JVM, Alvares V, Almeida DG, Thumé E, Thomaz EBAF, Queiroz RCS, Souza MR, Lein A, Lopes DP, Staton CA, Vissoci JRN, Facchini LA. Addressing geographic access barriers to emergency care services: a national ecologic study of hospitals in Brazil. Int $J$ Equity Health 2017; 16(1):149.

22. Torres SFS, Belisário AS, Melo EM. A rede de urgência e emergência da macrorregião norte de Minas Gerais: um estudo de caso. Saude soc. 2015; 24(1):361-373.

23. Duarte JG, Gomes SC, Pinto MT, Gomes MASM. Perfil dos pacientes internados em serviços de pediatria no município do Rio de Janeiro: mudamos? Physis 2012; 22(1):199-214.

24. Rehem TCMSB, Ciosak SI, Egry EY. Internações Sensíveis à Atenção Primária: usuários detectam falha no acesso aos serviços. Rev Esc Enferm USP [serial on the Internet]. 2014 [cited 2018 Jun 22]; 48(2):[about 6 p.]. Available from: http://www.scielo.br/pdf/reeusp/ v48nspe2/pt_0080-6234-reeusp-48-nspe2-00066.pdf

25. Cirino S, Gonçalves LA, Gonçalves MB, Cursi ES. Modelo não linear de localização de instalações de serviços de saúde com indicador de acessibilidade incorporado. Cad Saude Publica 2018; 34(3):1-12.

26. World Bank. Um ajuste justo: análise da eficiência e equidade do gasto público no Brasil. Washington: World Bank Group; 2017.

27. Pazin-Filho A, Almeida E, Cirilo LP, Lourençato FM, Baptista LM, Pintyá JP, Capeli RD, Silva SMPF, Wolf CM, Dinardi MM, Scarpelini S, Damasceno MC. Impacto de leitos de longa permanência no desempenho de hospital terciário em emergências. Rev Saude Publica [serial on the Internet]. 2015 [cited 2017 aug 6];49(83):[about 8 p.]. Available from: https://www. revistas.usp.br/rsp/article/download/130452/126862 
28. Braga Neto FC, Barbosa PR, Santos IS. Atenção hospitalar: evolução histórica e tendências. In: Giovanella L, Escorel S, Lobato, LVC, Noronha JC, Carvalho AI, organizadores. Politicas e sistemas de saúde no Brasil. Rio de Janeiro: Fiocruz, 2012. p. 577-608.

29. Cecílio LCO. A Atenção Básica à Saúde e a construção das redes temáticas de saúde: qual pode ser o seu papel? Cien Saude Colet [serial on the Internet]. 2012 [cited 2017 Apr 10]17(11):[about 9 p.]. Available from: http://www.scielo.br/pdf/csc/v17n11/v17n11a05.pdf

30. Almeida PF, Gérvas J, Freire JM, Giovanella L. Estratégias de integração entre atenção primária à saúde e atenção especializada: paralelos entre Brasil e Espanha. Saúde debate [serial on the Internet]. 2013 Sep [cited 2019 Apr 03];11(1): [about 10p.]. Available from: http://www.scielo.br/scielo.php?script=sci_arttext\&pid=S0103-11042013000300004\&lng=en

31. Caldeira AP, Fernandes VBL, Fonseca WP, Faria AA. Internações pediátricas por condições sensíveis à atenção primária em Montes Claros, Minas Gerais, Brasil. Rev. Bras. Saúde Mater. Infant. [serial on the Internet]. 2011 Mar [cited 2017 Sep 1];11(1):[about 10 p.]. Available from: http://www.scielo.br/pdf/rbs$\mathrm{mi} / \mathrm{v} 11 \mathrm{n} 1 / \mathrm{a} 07 \mathrm{v} 11 \mathrm{n} 1 . p d f$

32. Aguilera SLVU, França BHS, Moysés ST, Moysés SJ. Articulação entre os níveis de atenção dos serviços de saúde na Região Metropolitana de Curitiba: desafios para os gestores. Rev. Adm. Pública 2013; 47(4):10211039.

33. Santos AM, Giovanella L. Governança regional: estratégias e disputas para gestão em saúde. Rev Saude Publica 2014; 48(4):622-631.

Artigo apresentado em 15/08/2018

Aprovado em 05/06/2019

Versão final apresentada em 07/06/2019

Editores-chefes: Romeu Gomes, Antônio Augusto Moura da Silva 